\section{The effect of lens design on dysphotopsia in different acrylic IOLs}

S Shambhu, VA Shanmuganathan and SJ Charles

\begin{abstract}
Purpose To compare the incidence and severity of all grades of dysphotopsia in three types of acrylic intraocular lenses: the Acrysof MA30 BA and MA60 BM IOLs (Alcon) and the Akreos Fit one-piece IOL (Bausch and Lomb). Methods In all 111 patients were prospectively recruited who had undergone uncomplicated cataract surgery in the previous 12 months. Patients were assessed using a combination of a questionnaire and standardised provocation test, and were scored on a grading scale of 0-6 (0 indicating no glare and 6 indicating the most severe symptoms).

Results The overall incidence of dysphotopsia was $\mathbf{7 7 . 7 \%}$. Patients with absent or mild symptoms (scoring 2 or less on the grading scale) were greater in the group implanted with the Akreos IOLs (49/65 eyes, $75 \%)$ as opposed to (44/92 eyes, $48 \%$ ) of the eyes implanted with Acrysof lenses. Patients with more marked symptoms of glare as judged by scores of 5-6 were uncommon, but more prevalent in the Acrysof lenses (12/92 eyes, $13 \%$ ) compared to the Akreos lenses (3/65 eyes, $4.6 \%$ ) respectively. Statistically the Mann-Whitney test showed that there was significantly less dysphotopsia with the Akreos lens when compared to the Acrysof MA30 $(P=0.005)$ and MA60 lenses $(P=0.002)$.

Conclusion This study demonstrates that dysphotopsia symptoms are commonly seen in certain brands of Acrylic IOLs. However, differences in design (not only related to the edge) significantly reduce the incidence of moderate and severe grades of dysphotopic symptoms.
\end{abstract}

Eye (2005) 19, 567-570. doi:10.1038/sj.eye.6701568

Published online 24 September 2004

Keywords: dysphotopsia; glare; IOL; Akreos; Acrysof

\section{Introduction}

The advances in lens technology over recent years have seen the advent of the acrylic IOLs. The Acrysof IOL (Alcon) was introduced into the UK in 1996. These IOLs have been increasingly ${ }^{1}$ used and confer various advantages over the older lens designs particularly with regards to the rate of posterior capsule opacification (PCO) ${ }^{2}$ However, there have been well-documented associated symptoms of visually undesired phenomena. ${ }^{3}$ These phenomena may take many forms including glare and edge effects and have been collectively described as dysphotopsia. ${ }^{4}$ While newer acrylic lens types have been introduced there has been little work comparing dysphotopsia between different acrylic IOLs. At our institution we predominantly use both the Acrysof (Alcon) and the Akreos IOLs (Bausch and Lomb). The aim of this study was to assess and compare the dysphotopsia of all grades of severity in the Akreos fit 2-plate haptic and Acrysof MA30, and MA60 IOLs.

\section{Material and methods}

Following local ethics approval and informed consent, patients were recruited over a 7-week period from the outpatient department. Patients were chosen if they had undergone unilateral uncomplicated phacoemulsification surgery with in the bag IOL insertion and had obtained a postoperative vision of $6 / 9$ or better. The second unoperated eye was used as a control. Patients were excluded if they had ocular comorbidity associated with reduced postoperative visual acuity such as macular degeneration and PCO. PCO was excluded by slit-lamp examination.

The subject's age, pupil size, and time since surgery were noted in order to interpret any results correctly as these factors can affect the incidence of dysphotopsia. Patients were
Manchester Royal Eye Hospital

Manchester UK

Correspondence: SJ Charles Manchester Royal Eye Hospital,

Oxford Road, Manchester M13 9WH, UK

Tel: +441612765631

Fax: + 441612726618

E-mail: stephen.charles@

cmmc.nhs.uk

Received: 4 December 2003 Accepted: 12 March 2004

Published online:

24 September 2004

Presented in part as a poster at the Royal College of Ophthalmologists Annual Congress, Birmingham, UK, May 2003 
assessed using a protocol consisting of

a combined examination and questionnaire method.

This was carried out by a single investigator (SS) for consistency. The questions were phrased to elicit severity of dysphotopic symptoms to fit in with the grading scale shown in Table 1 . Scores ranged between 0 and 3, 0 indicating no symptoms of dysphotopsia and 3

indicating severe symptoms as indicated by the patient complaining of severe symptoms of dysphotopsia prior to specific questioning on the issue.

The examination consisted of a provocation, which was carried out under standardised conditions both in photopic (70 lux) and scotopic (5 lux) light using a light meter (ACT 1330 digital light meter manufactured by ACT meters LTD, St Helens, UK). Patients were asked to fixate on the top line of a Snellen chart at $3 \mathrm{~m}$. A slit beam (height $6.5 \mathrm{~mm}$, width $12 \mathrm{~mm}$ ) was shone at the pupil edge at $30^{\circ}$ and $60^{\circ}$ horizontal inclination. Patients were again scored using the grading scale shown in Table 1.

The total for the questions and the examination were added up with a possible range of scores between 0 and 6. These were subdivided into categories of severity. A score of zero indicated an absence of dysphotopsia and 1-2 (mild), 2-4 (moderate), and 5-6 (severe symptoms of dysphotopsia).

Table 1 Grading scale of dysphotopsia using questions and examination

\begin{tabular}{|c|c|c|}
\hline Grade & Response to question & Response to examination \\
\hline 0 & No dysphotopsia & No dysphotopsia \\
\hline 1 & $\begin{array}{l}\text { Minor amount of } \\
\text { dysphotopsia but only } \\
\text { when patient asked } \\
\text { if present }\end{array}$ & $\begin{array}{l}\text { Mild } \\
\text { Slight detection of } \\
\text { dysphotopsia effect }\end{array}$ \\
\hline 2 & $\begin{array}{l}\text { Patient confirms } \\
\text { significant dysphotopsia } \\
\text { on questioning }\end{array}$ & $\begin{array}{l}\text { Moderate } \\
\text { Definite dysphotopsia } \\
\text { effect noticed }\end{array}$ \\
\hline 3 & $\begin{array}{l}\text { Patient complains } \\
\text { of significant } \\
\text { dysphotopsia } \\
\text { prior to specific } \\
\text { questioning }\end{array}$ & $\begin{array}{l}\text { Severe } \\
\text { Unpleasant } \\
\text { dysphotopsia } \\
\text { Dazzling }\end{array}$ \\
\hline
\end{tabular}

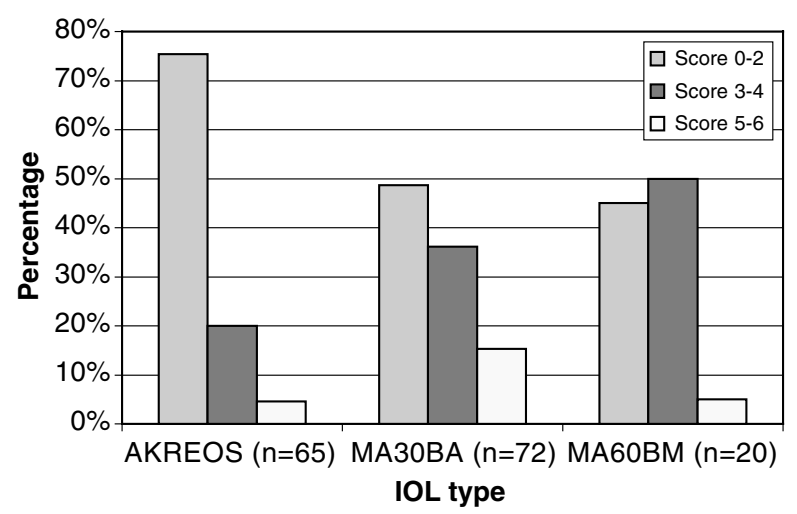

Figure 1 Graph illustrating \% frequency of grades of dysphotopsia vs IOL type.

\section{Results}

A total of 111 patients were recruited (222 eyes) and no patient invited to the study declined participation. The mean age of the Akreos, MA30BA, and MA60BM lens groups were $70.2,69.8$, and 69.9 years old respectively (mean 69.7 years). The average time of assessment from the date of surgery was $6.4,5.9$ and 6.1 weeks postoperatively for the Akreos, MA30, and MA60 IOL groups respectively (overall mean 6 weeks). The mean pupil size for the Akreos, MA30, and MA60 groups were 3.2, 3.1, and $3.3 \mathrm{~mm}$ respectively and $3.2 \mathrm{~mm}$ overall.

A total of 65 subjects were implanted with the Akreos IOL and 92 with the Acrysof IOL. A summary of the scores for each of the lens types is shown in Table 2 and illustrated in Figure 1. The average score was 1.56, 2.43, and 2.65 for the Akreos, Acrysof MA30, and MA60 IOLs respectively. In total, $75 \%$ (Akreos) as compared to $48 \%$ (Acrysof) had mild or no symptoms. Severe symptoms were uncommon (4.6\% for the Akreos and 13\% for the Acrysof). There was no difference in the patient's scores to the two different angles of incident light. All the results from the control eyes scored zero.

Statistical analysis was used to evaluate the significance of the differences in data obtained. The Mann-Whitney test was used as a standard nonparametric test to detect any significant difference between the three groups. Evaluation of the Akreos IOL against the Acrysof MA30BA IOL revealed that there was

Table 2 Results of number of patients with particular lens type $v$ s total score following assessment with the combined questioning and examination

\begin{tabular}{lrrrrrrrr}
\hline Total score & \multicolumn{1}{c}{1} & \multicolumn{1}{c}{2} & \multicolumn{1}{c}{3} & 4 & 5 & 6 & Total no of eyes \\
\hline Akreos & 18 & 18 & 13 & 8 & 5 & 3 & 0 & 65 \\
MA30BA & 15 & 11 & 9 & 15 & 11 & 9 & 2 & 72 \\
MA60BM & 2 & 1 & 6 & 5 & 5 & 1 & 0 & 20 \\
Total no of eyes & 35 & 30 & 28 & 28 & 21 & 13 & 2 & 157 \\
\hline
\end{tabular}


significantly less dysphotopsia in the Akreos group at the $5 \%$ level $(P=0.005)$. Comparison of the Akreos IOL against the Acrysof MA60BM gave similar results $(P=0.002)$. No statistically significant difference at the $5 \%$ level was demonstrated between the two Acrysof MA30BA and MA60BM IOLs $(P=0.526)$. All IOLs showed statistically significant differences when compared against control eyes $(P<0.001)$.

\section{Discussion}

From our study we have demonstrated that dysphotopsia is common in both the Akreos and Acrysof acrylic IOLs. However, there are more severe symptoms of dysphotopsia with both the Acrysof MA30BA and MA60BM IOLs when compared to the Akreos.

The severest symptoms of dysphotopsia can have a marked impact on a patient's postoperative visual rehabilitation. Davison catalogues 14 out of 6668 cases implanted with the Acrysof MA30 and MA60 where patients had complained vigorously of dysphotopic symptoms. ${ }^{5}$ In five cases this warranted an IOL exchange. In another study by Fabrowitz et $a l^{3}$ nine patients were identified over a 2-year period where symptoms were severe enough to warrant an IOL exchange with a subsequent improvement. From our study we reported 15 patients with severe dysphotopsia (scores of 5-6). While these patients complained of these symptoms (particularly that of glare), none of the cases were severe enough to justify an IOL exchange. The capacity to put up with symptoms outweighed the risks associated with further intraocular surgery. Furthermore, as Davison ${ }^{5}$ describes there can be an improvement in symptoms over time in some cases and as our assessment was carried out relatively soon after surgery it was decided that observation was the most appropriate course of management.

Comparative studies between IOL lens types have been carried out in the past. One study by Tester et al ${ }^{4}$ showed that $49 \%$ of patients reported some type of dysphotopsia. This study further concluded that there was significantly more dysphotopsia with the Acrysof IOLs when compared with five other lens types including silicone and PMMA IOLs. Hwang et $a l^{6}$ approached the assessment of dysphotopsia by a different angle by looking specifically at patient satisfaction through a telephone survey. Interestingly, the MA60 and the silicone SI-3/ SWI-40 performed better than the MA30 in terms of glare vision. This contrasts with our work where there was no difference between the two types of Acrysof IOLs.

While these studies broadly agree with our own conclusions regarding the Acrysof IOL they highlight the difficulties in interpreting dysphotopsia as a symptom and thus making direct comparisons problematic. There is no standardised questionnaire or universally accepted grading system. Much depends on the phrasing of questions asked and the patients' subjective responses. To simplify our assessment we did not subdivide the dysphotopsia into any subtypes or ask about any specific activities that have been affected (e.g. night driving) as in other studies. With our method of assessment we wanted to introduce an element of objectivity. By devising a simple and reproducible examination technique we were able to standardise the conditions. Thus, we examined the effect of a point light source to provoke dysphotopic symptoms although we accept that the patient responses were still subjective.

The overall incidence of dysphotopsia of all severities in this study was high at $77.7 \%$ of patients. This in part can be explained by the square and sharp edged designs of these IOLs. ${ }^{7}$ These designs are pivotal in the prevention of PCO by preventing lens epithelial cell migration. ${ }^{8}$ However, ray tracing techniques have indicated that there is greater internal light scattering onto the retina from the sharp IOL edges than those with rounded edges. ${ }^{9}$ Recent works, which has realised the importance of the edge surface, have used a textured edge design of the Acrysof IOL with marked improvements in postoperative glare. ${ }^{10}$ Since the completion of our work Alcon have introduced newer versions of the Acrysof range (MA30AC and MA60AC IOLs) that have frosted edges.

Other factors other than the edge play a role in creating symptoms of dysphotopsia. The MA30 and MA60 have a loop angulation of $5^{\circ}$ and $10^{\circ}$ respectively. In comparison, the Akreos lens angulation is $0^{\circ}$. The Acrysof has an unequal biconvex optic with a greater radius of curvature on the anterior surface $(32 \mathrm{~mm}$ anterior and $16 \mathrm{~mm}$ posterior) as opposed to the Akreos lens, which is equi-biconvex $(15 \mathrm{~mm}$ anterior and posterior). The effects of surface convexity have been studied experimentally using an artificial eye model and optical analysis computer software. ${ }^{11}$ This work demonstrated that the unequal bi-convex design produced internal reflections that were focussed on an area 60 times smaller than that of an equi-convex design. This leads to a far more concentrated light image. This would manifest itself clinically by the increased potential for patients to experience postoperative glare. This study also showed that the light reflected from the anterior surface of the lens increases with increasing refractive index. The Acrysof refractive index is 1.56 as opposed to the Akreos 1.46. Further work ${ }^{12}$ by the same group using similar methodology suggested that IOLs with anterior radius of curvature of $17 \mathrm{~mm}$ or less will minimise surface reflections. 
Thus, our study demonstrates that the combination of an unequi-biconvex design and higher refractive index of the Acrysof IOL may explain some of our clinical findings.

Without a previous history of postoperative dysphotopsia, it can be difficult to predict which patients are likely to develop dysphotopsia as it is clearly multifactorial. There are both patient and IOL factors to consider. Patient's perception and tolerance of symptoms as well as intrinsic factors such as an individual's corneal power ${ }^{11}$ have a role to play. This may explain the variability of the range of scores that we encountered.

We conclude that different brands of Acrylic IOLs display varying degrees of dysphotopic symptoms. However, there are several lens design factors such as edge shape, refractive index, and shape of the anterior surface that can influence and ultimately reduce the severity of dysphotopic phenomena. With the current climate of increasing patient expectations coupled with the goal of achieving long-term patient satisfaction, the ability to have low levels of glare and edge effect must be considered in the future selection and development of IOLs.

\section{References}

1 Leaming DV. Practice styles and preferences of ASCRS members- 2000 survey. American Society of Cataract and Refractive Surgery. J Cataract Refract Surg 2001; 27: 948-955.
2 Hayashi H, Hayashi Y. Quantitative comparison of PCO after PMMA, silicone and acrylic IOL implantation. Arch Ophthalmol 1998; 116: 1579-1582.

3 Farbowitz MA, Zabriskie NA, Crandall AS, Olson RJ, Miller KM. Visual complaints associated with the Acrysof acrylic intraocular lens. J Cataract Refract Surg 2000; 26: 1339-1345.

4 Tester R, Pace NL, Samore M, Olson RJ. Dysphotopsia in phakic and pseudophakic patients: incidence and relation to intraocular lens type. J Cataract Refract Surg 2000; 26: 810-816.

5 Davison J. Positive and negative dysphotopsia in patients with acrylic intraocular lenses. J Cataract Refract Surg 2000; 26: 1346-1355.

6 Hwang IP, Olson RJ. Patient satisfaction after uneventful cataract surgery with implantation of a silicone or acrylic foldable intraocular lens. J Cataract Refract Surg 2001; 27: 1607-1610.

7 Ellis M. Sharp-edged intraocular lens design as a cause of permanent glare. J Cataract Refract Surg 2001; 27: 1061-1064.

8 Nishi O, Nishi K. Preventing lens epithelial cell migration using intraocular lenses with sharp rectangular edges. J Cataract Refract Surg 2000; 27: 608-613.

9 Holladay JT, Lang A, Portney V. Analysis of edge glare phenomena in intraocular lens designs. J Cataract Refract Surg 1999; 25: 748-752.

10 Meacock WR, Spalton DJ, Khan S. The effect of texturing the intraocular lens edge on postoperative glare. Arch Ophthalmol. 2002; 120: 1294-1298.

11 Erie J, Banhauer M. Analysis of postoperative glare and intraocular lens design. J Cataract Refract Surg 2001; 27: 614-621.

12 Erie J, Banhauer M. Intraocular lens surfaces and their relationship to post operative glare. J Cataract Refract Surg 2003; 29: 336-341. 